\title{
Blue-Noise Sigma-Delta Modulator: Improving Substrate Noise and Nonlinear Amplifier Gain Effects
}

\author{
Eric C. Moule, Zeljko Ignjatovic \\ Dept. of Electrical and Computer Engineering \\ University of Rochester \\ Rochester, NY USA \\ \{ermoule; ignjatov\}@ece.rochester.edu
}

\begin{abstract}
This paper presents a method to reduce the effects of substrate noise and nonlinear amplifier gain on sigma-delta $(\Sigma \Delta)$ modulators through the use of blue-noise modulation. In addition to reducing the effects of substrate noise and nonlinear amplifier gain, the proposed architecture also suppresses the effects of integrator opamp non-idealities, such as 1/f noise and DC offset, and DAC DC offset and even-order nonlinearities. The architecture proposed herein is referred to as the blue-noise $\Sigma \Delta$ modulator. An example utilizing a thirdorder blue-noise $\Sigma \Delta$ modulator with a 1-bit quantizer is presented. Additionally, a method to generate the required blue-noise modulation sequence is proposed. Simulation results demonstrate that this architecture achieves an $8.8 \mathrm{~dB}$ improvement in SNR over the traditional third-order $\Sigma \Delta$ modulator.
\end{abstract}

\section{INTRODUCTION}

As CMOS technology scaling continues to reduce feature size, chip designers will be integrating more and more analog and digital circuitry together on the same die in an effort to reduce cost. However, as pointed out in [1] and [2], integration of systems-on-a-chip (SoC) requiring the placement of noise-sensitive analog blocks and noisy digital signal processing blocks together on a common substrate will most likely continue to increase the effect of substrate noise generated by the digital circuitry. In particular, mixedsignal designs such as $\Sigma \Delta$ ADCs, where the analog and digital components cannot necessarily be placed far from each other, will see significant performance degradations due to substrate noise. According to [3], the SNDR of a $\Sigma \Delta$ modulator may decrease by over $20 \mathrm{~dB}$ in the presence of noisy digital circuitry such as toggling inverters.

$\Sigma \Delta$ modulator performance is also adversely affected by non-ideal integrator opamp DC gain factors, in particular finite DC gain and nonlinear DC gain. The effect of finite DC gain on $\Sigma \Delta$ modulator performance has been well documented in literature to produce integrator leakage in which only a fraction of the previous output sample is added to the current input sample. This affects the extent to which the $\Sigma \Delta$ modulator shapes low-frequency quantization noise [4]. However, the effects of nonlinear DC gain on $\Sigma \Delta$ modulator performance have been largely overlooked until recently [5]. According to [5], nonlinear DC gain introduces harmonic distortions into the modulator output spectrum and also increases the in-band noise floor. This is further examined in Section II.

It is well known that $\Sigma \Delta$ ADCs are suitable for high resolution and low-to-moderate bandwidth applications. However, when circuit nonidealities such as substrate noise and nonlinear amplifier gain are introduced into the $\Sigma \Delta$ modulator, dynamic range is sacrificed. To remedy this, the blue-noise $\Sigma \Delta$ modulator architecture presented herein utilizes blue-noise modulation, whereby a signal with bluenoise spectral characteristics, used as a chopper modulation signal, eliminates the substrate noise and nonlinear amplifier gain spectral peaks by spreading them as noise across the entire modulator spectrum. This noise, along with the resulting quantization noise, is then shaped away from the baseband input signal to high frequency as is typical for oversampling $\Sigma \Delta$ modulators. The proposed method eliminates the strong distortion component near DC created by harmonics of the digital sampling clock and the evenorder harmonics produced by the nonlinear amplifier gain.

\section{NONLINEAR AMPLIFIER DC GAIN}

Non-ideal finite integrator amplifier DC gain results in integrator leakage, whereby only a fraction of the previous output sample is added to the current input sample. The transfer function representing a leaky integrator is given in Eq. (1), where $\alpha=1-\mathrm{A}_{0}^{-1}$ is defined as the integrator leakage factor $\left(\mathrm{A}_{0}\right.$ is the amplifier $\mathrm{DC}$ gain).

$$
H(z)=\frac{1}{1-\alpha z^{-1}}
$$

However, the amplifier DC gain does not remain constant over the range of all amplifier output values due to the change in the output transistors' output resistance [5]. In reality where large-signal swings are common at the amplifier output, the amplifier DC gain is a function of the amplifier output voltage, $v_{\text {out }}$. 


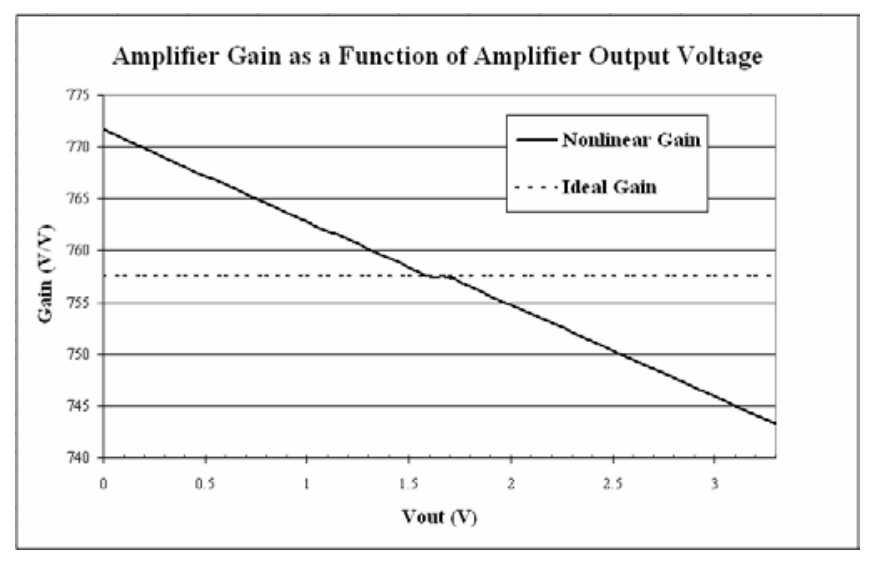

Figure 1. Two-stage amplifier DC gain as a function of $v_{\text {out }}$.

In particular, an amplifier with an NMOS output stage will have higher DC gain for lower amplifier output voltages, and the DC gain will decrease as the amplifier output voltage increases. This was verified through simulation of a twostage amplifier utilizing an NMOS common-source output stage with a PMOS active load. The resulting DC gain as a function of the output voltage for the two-stage amplifier is shown in Figure 1. The amplifier nonlinear DC gain characteristic causes the overall integrator gain and corresponding pole to constantly change as a function of the output voltage value [5]. Since the integrator gain is a function of the output value and output value depends on the previous input signal samples (i.e., integrator's gain depends on the input signal), the integrator exhibits non-linear behavior and harmonic distortions are introduced into the modulator output.

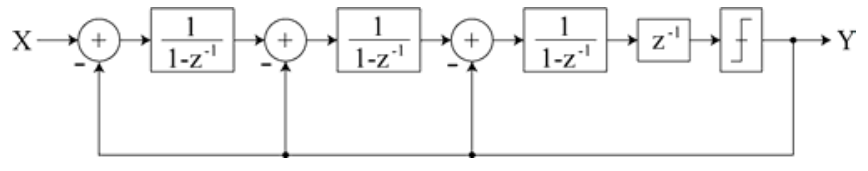

Figure 2. Traditional third-order $\Sigma \Delta$ modulator.

\section{Blue-Noise $\Sigma \Delta$ Modulator Design}

The blue-noise $\Sigma \Delta$ modulator is obtained by placing pairs of blue-noise multipliers before and after each non-delayed integrator in a traditional $\Sigma \Delta$ modulator, such as the one shown in Figure 2. In the case of a sample-delayed integrator, the integrator is first separated into a non-delayed integrator followed by the delay element, as shown by the final integrator in the cascade of integrators with feedback structure depicted in Figure 2. The multiplying sequence, $\mathrm{e}$ [n], used to modulate the signals throughout the blue-noise $\Sigma \Delta$ modulator is a sequence of 1's and -1's that has bluenoise spectral characteristics (i.e., the spectrum has a low frequency deficiency).

Identical blue-noise sequences are introduced into the modulator structure in pairs, thus they effectively perform multiplication by 1 , leaving the overall modulator function unchanged. Once the blue-noise multipliers are inserted, as shown in Figure 3a, one multiplier from each pair is moved through the modulator to produce the blue-noise $\Sigma \Delta$ modulator structure shown in Figure 3c. Figure $3 b$ shows the intermediate stage in rearranging the blue-noise multipliers to create the blue-noise $\Sigma \Delta$ modulator.

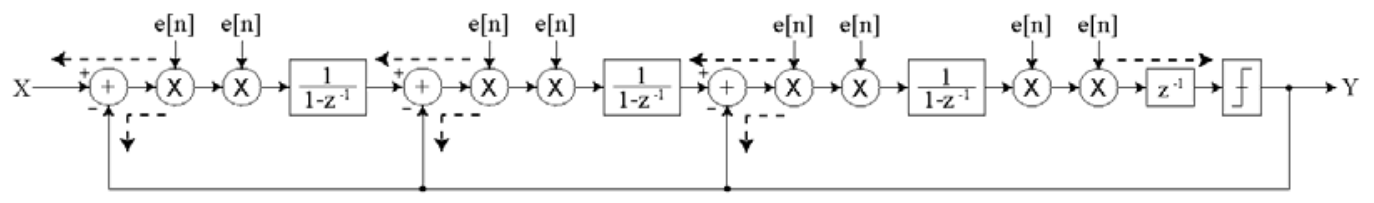

(a)

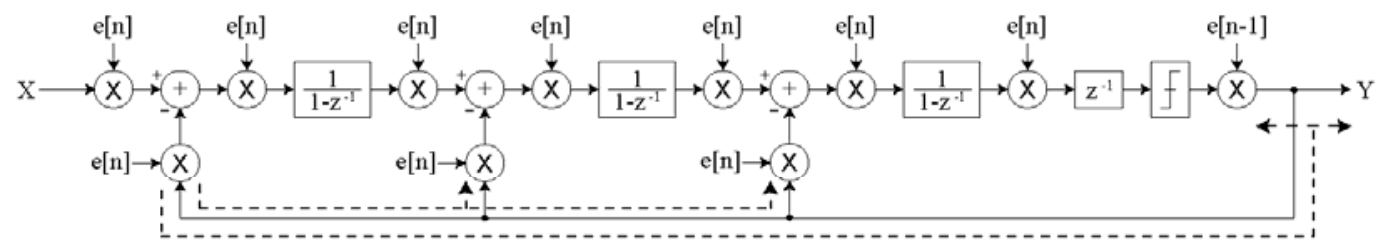

(b)

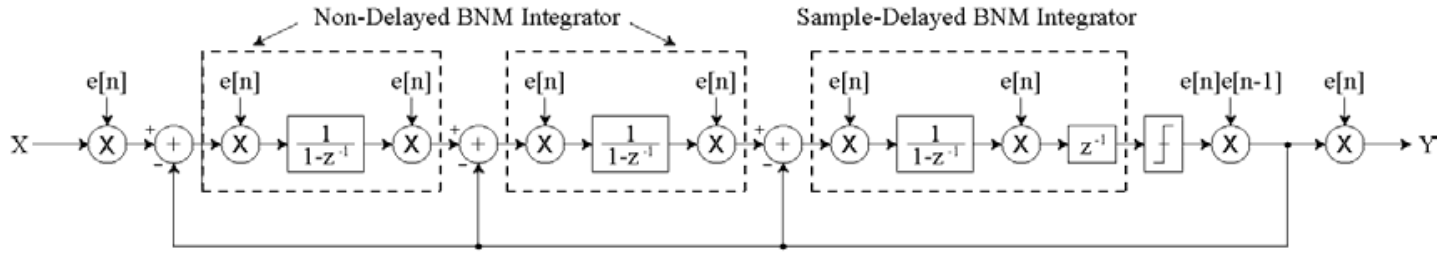

(c)

Figure 3. (a) Third-order $\Sigma \Delta$ modulator with blue-noise multiplier pairs added. (b) Third-order $\Sigma \Delta$ modulator with blue-noise multipliers rearranged. (c) Third-order blue-noise modulated $\Sigma \Delta$ modulator. 

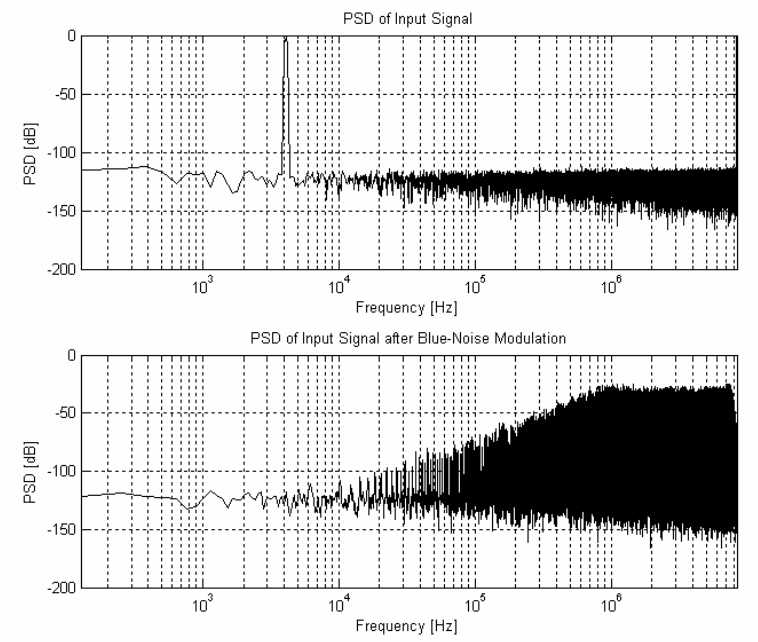

Figure 4. Spectrum of input signal before (top) and after (bottom) bluenoise modulation at the $\Sigma \Delta$ modulator input.

The dashed arrows in Figures $3 \mathrm{a}$ and $3 \mathrm{~b}$ depict the direction in which a particular blue-noise multiplier is moved. When the blue-noise modulation signal, e $[\mathrm{n}]$, is pushed through the delay element, $\mathrm{z}^{-1}$, the resulting modulation signal is the delayed blue-noise sequence, e[n-1]. The structural transformation described here may be applied to any traditional $\Sigma \Delta$ modulator to create a blue-noise $\Sigma \Delta$ modulator.

The blue-noise $\Sigma \Delta$ modulator architecture presented in Figure $3 \mathrm{c}$ incorporates the non-delayed blue-noise modulated (BNM) integrator and the sample-delayed BNM integrator structures. These structures take on the same architecture as the mirrored integrator structures proposed in [6]. A distinct advantage of the proposed blue-noise $\Sigma \Delta$ modulator is that it is not a major design digression from mature $\Sigma \Delta$ modulator technology.

In addition to spreading substrate noise and nonlinear amplifier gain, which exhibits strong spectral peaks, bluenoise modulation also negates the effects of integrator opamp and DAC non-idealities (1/f noise, DC offset, and even-order non-linearities). This is due to the fact that blue-noise modulation shapes the input signal about the Nyquist frequency before it sees the non-idealities from the integrator opamps and DAC. The input sinusoidal signal spectra before and after blue-noise modulation are shown in Figure 4. The undesirable noise contributions will remain at low frequency where the quantization noise is shaped by the blue-noise modulated integrator structures. When the output signal experiences the final blue-noise multiplier, the input signal is returned to baseband, while the shaped quantization noise along with the low frequency $1 / \mathrm{f}$ noise and DC offsets are pushed toward high frequency. This procedure is much like that described in [7] for the mirrored-integrator $\Sigma \Delta \mathrm{ADC}$.

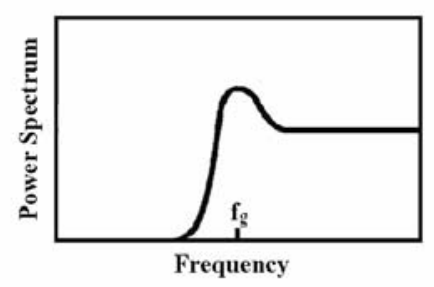

Figure 5. Blue-noise spectrum.

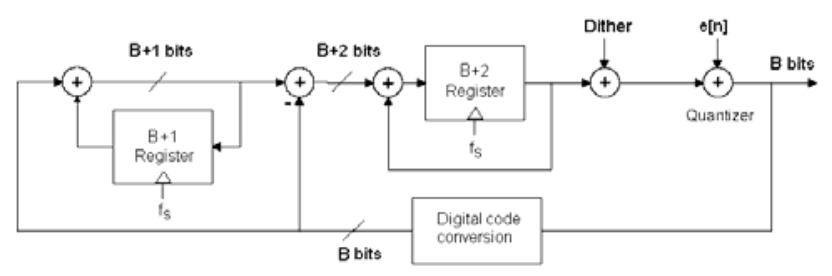

Figure 6. All-digital $\Sigma \Delta$ modulator.

\section{Blue-Noise SEQUence Generation}

The spectrum of a blue-noise sequence consists of low frequency deficiencies and uncorrelated high-frequency fluctuations that are classified as high-frequency white noise. The sharp transition between the energy limited low frequency portion of the blue-noise spectrum and the high frequency white noise occurs at the principal frequency. The principal frequency for a particular blue-noise sequence is commonly denoted $f_{\mathrm{g}}$. The spectrum of a typical blue-noise sequence is shown in Figure 5 [8].

The binary blue-noise sequence necessary for blue-noise modulation can be generated using an all-digital $\Sigma \Delta$ modulator. Replacing the integrators from traditional $\Sigma \Delta$ modulators with accumulators creates the all-digital $\Sigma \Delta$ architecture, which is shown in Figure 6. Since the bluenoise sequence used in the blue-noise $\Sigma \Delta$ modulator consists only of 1's and -1 's, the quantizer in the all-digital $\Sigma \Delta$ modulator needs to resolve $\mathrm{B}=1 \mathrm{bit}$. Similar algorithms that produce blue-noise sequences have been proposed for fractional-N PLL applications [9] and for DAC dynamicelement matching in multi-bit $\Sigma \Delta \mathrm{ADC}$ applications [10].

\section{Simulation Results}

The behavioral simulation results presented here were obtained from Matlab/Simulink models of a third-order, 1-bit $\Sigma \Delta$ modulator. The input used for the simulations was a sinusoidal signal with frequency $f_{\text {in }}=4.1 \mathrm{kHz}$ lying within a $32.8 \mathrm{kHz}$ bandwidth. The OSR was selected to be 128 , resulting in a sampling frequency of $f_{s}=8.397 \mathrm{MHz}$. The substrate coupling noise used for the simulations was obtained in the lab from Maxim IC's MAX1403 18-bit $\Sigma \Delta$ ADC. The measured noise, which contained the digital sampling clock and several strong higher harmonics and subharmonics, was imported into Matlab for the simulations and 


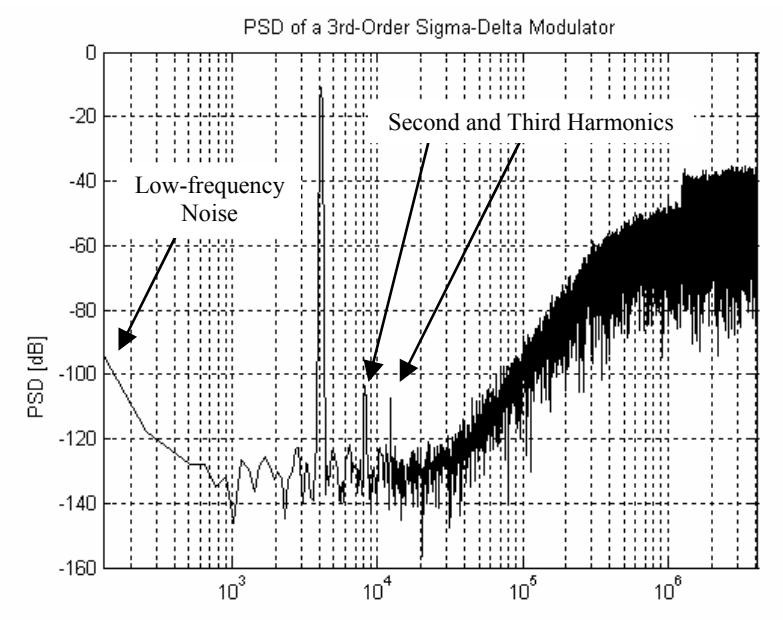

Figure 7. Output spectrum of third-order, 1-bit $\Sigma \Delta$ modulator.

injected into the first integrator in the $\Sigma \Delta$ modulator. Additionally, the nonlinear amplifier DC gain results presented in Section II are included in the model for the first integrator. In order to provide realistic simulations, non-ideal amplifier models were used for the integrators. The simulations were performed assuming room temperature, a finite amplifier gain, finite gain-bandwidth, slew rate, and saturation. In addition to the amplifier non-idealities, clock jitter was also taken into account.

The output spectrum of a typical third-order 1-bit $\Sigma \Delta$ modulator is shown in Figure 7. The output spectrum clearly depicts the modulator's vulnerability to low-frequency noise as well as even- and odd-order harmonics from the nonlinear amplifier gain, which limits the SNR to $76.6 \mathrm{~dB}$ in simulation. The output spectrum of the blue-noise $\Sigma \Delta$ modulator, shown in Figure 8, reveals that this architecture suppresses the low-frequency distortions near DC as well as the even-order harmonics created by the nonlinear amplifier gain. The resulting SNR for the blue-noise modulated architecture using the same parameters as the simulation without blue-noise modulation is $85.4 \mathrm{~dB}$. The proposed architecture demonstrates an $8.8 \mathrm{~dB}$ improvement in SNR from the traditional $\Sigma \Delta$ modulator design, which translates to an increase in effective resolution of 1.2 bits.

\section{CONCLUSION}

The blue-noise $\Sigma \Delta$ modulator has been proposed and described. The proposed design is not a major design digression from mature conventional $\Sigma \Delta$ modulator technology. Simulations of this architecture have proven the ability to suppress the effects of substrate noise generated by noisy digital blocks placed on the same die as sensitive analog circuits and nonlinear amplifier gain created by nonsymmetrical device properties. In addition to attenuating substrate noise and even-order nonlinear amplifier gain spectral peaks, the proposed design eliminates $1 /$ f noise, DC

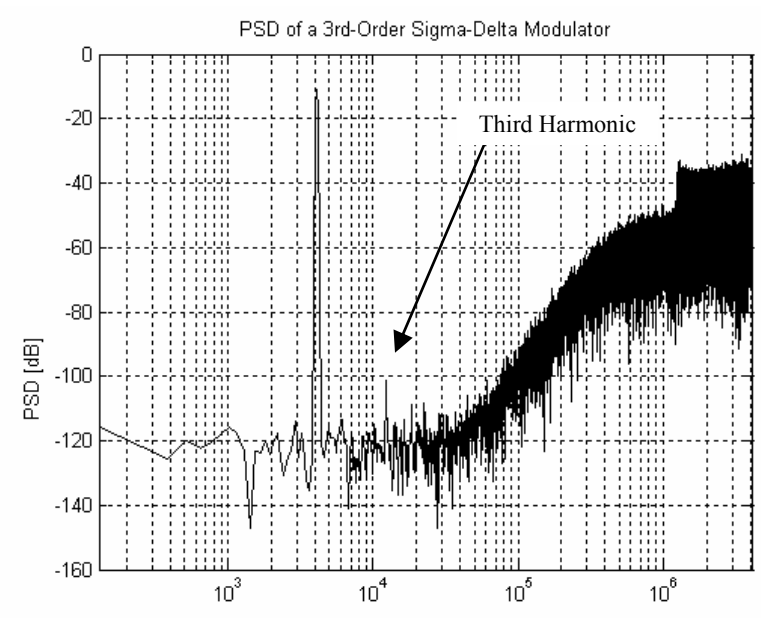

Figure 8. Output spectrum of third-order, 1-bit blue-noise $\Sigma \Delta$ modulator.

offset, and even order non-linearities. For the 1-bit, thirdorder blue-noise $\Sigma \Delta$ modulator with an OSR of 128, an overall improvement in SNR of $8.8 \mathrm{~dB}$ has been demonstrated. In an ongoing effort, we plan to demonstrate the effectiveness of the blue-noise modulation in higherorder low-pass and band-pass $\Sigma \Delta$ modulator prototypes.

\section{REFERENCES}

[1] P. Heydari, "Characterizing the effects of the PLL jitter due to substrate noise in dicrete-time delta-sigma modulators," IEEE Trans. Circuits Syst. I, Fundam. Theory Appl., vol. 52, no. 6, pp. 1073-1085, June 2005.

[2] Y. Zinzius, G. Gielen, W. Sansen, "Analyzing the impact of substrate noise on embedded analog-to-digital converters," in Proc. IEEE International Conference on Circuits and Systems for Communications, June 2002, pp.82-85.

[3] M.S. Peng, H.S. Lee, "Study of substrate noise and techniques for minimization," IEEE J. Solid-StateCircuits, vol. 39, no. 11, pp. 20802086, November 2004.

[4] S. Rabii, B.A. Wooley, The Design of Low-Voltage, Low-Power Sigma-Delta Modulators, Kluwer Academic Publishers, 1999.

[5] H. Zare-Hoseini, I. Kale, "On the effects of finite and nonlinear DCgain of the amplifiers in switched-capacitor $\Delta \Sigma$ modulators," in Proc. ISCAS, 2005, pp. 2547-2550.

[6] Z. Ignjatovic, M. Bocko, "A fully-differential switched capacitor chopper stabilized high-pass filter (mirrored integrator)," in Proc. ISCAS, 2004, pp. 857-860.

[7] Z. Ignjatovic, M. Bocko, "Sigma-delta analog to digital converter architecture based upon a modulator design employing a mirrored integrator," in Proc. ISCAS, 2004, pp. 1132 -1135.

[8] R.A. Ulichney, "Dithering with Blue Noise," in Proc. IEEE, vol. 76, pp. 56-79, Jan. 1988.

[9] M.Kozak, E.G. Friedman, "Design and simulation of fractional-N PLL frequency synthesizers," in Proc. ISCAS, 2004, pp. 780-783.

[10] T.L. Brooks, D.H. Robertson, D.F. Kelly, A. Del Muro, S.W. Harston, "A cascaded sigma-delta pipeline A/D converter with 1.25 MHz signal bandwidth and $89 \mathrm{~dB}$ SNR," IEEE J. Solid-StateCircuits, vol. 32, no. 12, pp. 1896-1906, December 1997. 